\title{
Feasibility Study Wear Rate Measurement Using 3D Scan Imaging
}

\author{
Kurniawan $^{1}$, Rochim Suratman $^{1}$, Bagus Budiwantoro ${ }^{1}$, Satryo S. Brodjonegoro ${ }^{1}$, and Riona I. Media ${ }^{2}$
}

\begin{abstract}
This paper studied on optimizing the 3D scan parameters of flared chisel bucket teeth as a worn component to achieve prospect scanning micro wear in different percentage of volume loss. Taguchi L8 orthogonal array was used to design of experiment. Specimen composed in three different volume loss $1 \%, 3 \%$, and $5 \%$ were scanned to obtain model CAD volumes. Volume dissimilarity was calculated by comparing model CAD volume and mass - density conversion. Shutter Time, grid, distance, and meshing were chosen as 3D scan parameters. Biggest effect and optimal scanning parameter were determined using the signal-to-noise $(S / N)$ ratio which was calculated for volume dissimilarity according to the "thesmaller-the-better' approach. The effects of scanning parameter were evaluated by analysis of variance. 3D imaging feasibility study for small volume measurement could be a critical point in micro wear measurement. The statistical analysis indicated that the parameters that have the biggest effect on volume dissimilarity with $1 \%, 3 \%$, and $5 \%$ volume loss are the meshing method and distance, respectively. The optimal setting parameter capturing image is 12 ms shutter time with normal meshing above 15 mm distance. Additionally, the interaction scanning parameter for $1 \%, 3 \%$, and $5 \%$ volume loss was presented.
\end{abstract}

Keywords-3D Scan, Wear, Measurement.

\section{INTRODUCTION}

Research on the wear resistant material has been developed to ensure service operation by increasing the lifetime of exploitation component. A study in wear measurement recently shows between laboratory work and in situ measurement had obvious variance. However, the main handicap in laboratory work is a limitation of its representation about the real situation. In situ measurement should be carried out considering validity, uncomplicated and cost respectively [1][2], and [3]. Recently there is no appropriate method or device in wear measurement mainly on site service. With this intention, preliminary study considering $3 \mathrm{~d}$ scan imaging in wear measurement should be proposed.

In the previous study, wear rate measurement of wear resistance component in a large number had carried out periodically during service operation. Several measurement techniques had applied even barely using a common ruler steel at the static position as a scale. Observation involved time-consuming for disassembled and weighing. This imprecise and costly measurement lead to an enormous fail

\footnotetext{
${ }^{1}$ Kurniawan, Rochim Suratman, Bagus Budiwantoro, and Satryo S. Brodjonegoro are with Department of Mechanical Engineering, Institut Teknologi Bandung, Bandung, Indonesia. E-mail: iwan21368@gmail.com.

${ }^{2}$ Riona I. Media is with Department of Design Engineering, Politeknik Manufaktur Negeri Bandung, Bandung, Indonesia.
}

result. Consequently, it is necessary to develop a simple method to measure the wear rate with a high accuracy but less time-consuming in the measurement process. A study in improvement wear measurement method using 3D scan could be prized in providing micro wear measurement tool [4]. This paper studied optimizing the 3D scan parameters worn component to achieve prospect scanning micro wear in different percentage of volume loss.

In metrology, precision refers to measurement dispersion. The measurement error (the mean) can be close to zero even if the system is not very precise (but it needs a good trueness). In other words, the less scattered the measurement data the more precise the equipment. A formal definition of precision is the closeness of agreement between indications or measured quantity values obtained by replicate measurements on the same or similar objects under specified conditions [5]. The word trueness rather gives information on the difference between the mean of measurements and the real dimension regardless of dispersion. In other words, the closest the mean of measurements is to the nominal value, the more trueness the equipment has. A formal definition of trueness is the closeness of agreement between the average of an infinite number of replicate measured quantity values and reference quantity value.

Furthermore, the study should show feasibility $3 \mathrm{~d}$ scan imaging to measure any micro wears and calculate its loss weight or wear rate respectively [6]. In the present study, meshing level was made considering to elaborate 3D model generation. In addition, smooth meshing level was taken generally to provide an accurate model. It should be elaborate in wear measurement application whether this particular factor [5]. Although 3D scan technology today provides 20100 micron meter accuracy and 10-20 micron meter for the advanced one, there is no appropriate method or device in wear measurement mainly on-site service which still lacks of a comparative study for their actual precision and robustness. This research proposes an effective approach in preliminary study considering 3D scan imaging in wear measurement validated with Taguchi experimental based method to obtain the optimal setting parameters.

\section{METHODOLOGY}

The proposed approach is integrating the design of experiments by means of the Taguchi method with CAD software object-oriented to obtain the best setting parameters in scanning images of wear on the specimens. First of all, twelve specimens are prepared for selecting the parameters according to the L8 orthogonal array, then they are scanned and extracted to acquire the mesh levels. In addition to confirm 3D CAD data from the 3D scan, rough mesh model must be aligned and merged then compared to $3 \mathrm{D}$ model on 
the CAD software as shown in Fig.1. As a result, the independent different values and volume scanned are designated as a final response.

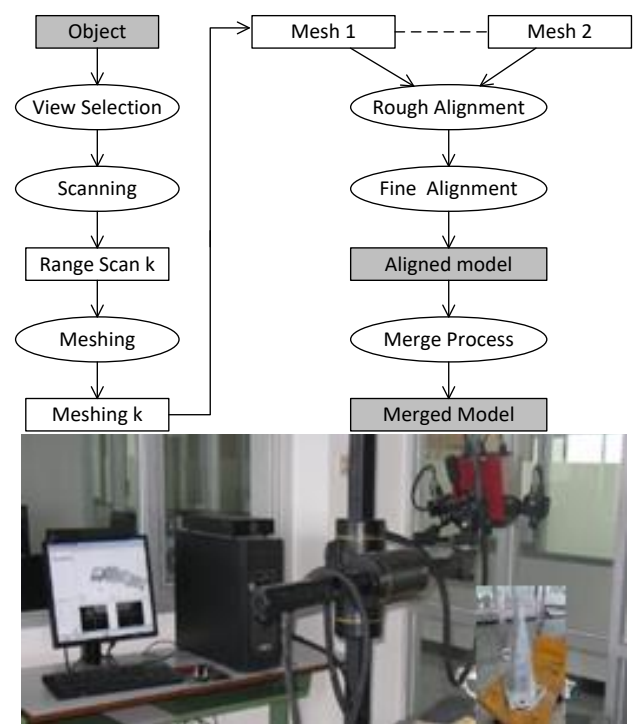

Figure 1. The pipeline of the model reconstruction (top) 3D scan apparatus GOM ATOS (bottom).

\section{A. Taguchi Method}

Taguchi method is used in the industry to decrease the product development period for the design and production which also decrease the costs and increase the profit of the company. Taguchi method also allows controlling the variations caused by the uncontrollable factors which are not taken into consideration at the conventional design of experiment Taguchi converts the objective function values to signal-to-noise $(\mathrm{S} / \mathrm{N})$ ratio for measure the performance characteristics of the levels of control factors against these factors. $\mathrm{S} / \mathrm{N}$ ratio is defined as the desired signal ratio for the undesired random noise value and shows the quality characteristics of the experimental data. There are three different functions used which are known as the objective function and also defined as $\mathrm{S} / \mathrm{N}$ ratio: "the-larger-thebetter", "the-smaller-the-better" and "the-nominal-thebest'. Besides, ANOVA is used to determine the statistical significance of the cutting parameters. The optimum combination of the cutting parameters is determined with the help of ANOVA and $\mathrm{S} / \mathrm{N}$ ratios. Lastly, confirmation experiments are done using the optimum machining parameters which were found by the Taguchi optimization method and thereby validation of the optimization is tested [7].

In this study, 3D scan parameters are optimized for model CAD volumes occurred for $1 \%, 3 \%$, and $5 \%$ volume loss. Shutter time, grid, distance, and meshing are chosen as 3D scan parameters as scanning parameters (variables). The parameter levels were chosen within the intervals recommended by the $3 \mathrm{D}$ scan manufacturer. An L8 orthogonal array of Taguchi method was used in the design of the experiment. Variables and their levels are given in Table 1.
TABLE 1.

VARIABLES AND THEIR LEVEL

\begin{tabular}{clll}
\hline \multirow{2}{*}{ Designation } & \multirow{2}{*}{ Process parameters } & \multicolumn{2}{c}{ Levels } \\
\cline { 3 - 4 } & & Low & High \\
\hline$A$ & Shutter Time, $(\mathrm{ms})$ & 8 & 12 \\
$B$ & Grid $(\mathrm{mm})$ & 15 & 35 \\
$C$ & Distance $(\mathrm{mm})$ & 50 & 80 \\
$D$ & Mesh $(-)$ & 100 & 50 \\
\hline \hline
\end{tabular}

Optimization of the scanning parameters has been performed separately for specimens with $1 \%, 3 \%$, and $5 \%$ volume loss. The smaller-the-better performance characteristic for dissimilarity was applied in order to obtain the optimal scanning parameters. $\mathrm{S} / \mathrm{N}$ ratio $\mathrm{g}$ is defined as follows:

$$
S / N=-\log 10 \frac{1}{n} \sum_{i-1}^{n} Y_{i}^{2}
$$

Where $\mathrm{Yi}$ is the observed data in the experiment and $\mathrm{n}$ is the number of experiments. The $\mathrm{S} / \mathrm{N}$ ratios of four factors using Eq. (1) were calculated for dissimilarity. ANOVA was applied with $95 \%$ confidence level to determine the significance level of the variables on dissimilarity. Optimization process based on the Taguchi method was performed by Minitab 17 software. Minitab is a powerful software that solves many statistical problems with ease. This software is frequently used performing statistical analysis and quality improvement in the area of mathematics, statistics, economics, sports, and engineering.

\section{B. 3D Scan for Model CAD Reconstruction}

Specimen composed in three different volume loss $1 \%, 3 \%$, and $5 \%$ were scanned to obtain model CAD volumes. Volume dissimilarity was calculated by comparing model CAD volume and mass - density conversion. Management of scanned data taking into consideration alignment tools and merge tools. The comparison will be based on the scanning and reconstruction of several worn components with a variety of scar and form objects. Experiment using 3D scan imaging GOM ATOS and the quality of the reconstructed 3D models is evaluated using Geomagic software. Model reconstruction procedure refers to the pipeline of model reconstruction. Model reconstruction converted to CAD model to obtained its volume [8]. The volume difference between the volume reconstruction model and actual specimen elevated dissimilarity. Dissimilarity $\psi$ defined as ratio volume scan obtained $v_{s}$ to volume mass conversion $v_{c}$ as follows:

$$
\psi=100-\left(v_{s} / v_{c}\right)
$$

In Taguchi method, optimization is verified by confirmation experiments after the determination of the variable levels that will give the optimal results. This method of calculating CI is the traditional statistical approach.

$$
C I_{\% \operatorname{dissV} L}=\sqrt{F_{\alpha}\left(1, f_{e}\right) V_{e}\left[\frac{1}{n_{e f f}}+\frac{1}{R}\right]}
$$

Where $F_{\alpha}\left(1, f_{e}\right)$ is the F-ratio at the confidence level of (1$\alpha$ ) against the degree of freedom (DoF) 1 and error DoF $f_{e}$. R is the sample size for conformation experiments; $V_{e}$ is error variance; $n_{e f f}=(N /(1+D o F)), \mathrm{N}$ is a total number of trials; and DoF is a total degrees of freedom associated with 
the estimate mean value. The $\mathrm{F}$ ratio is determined from the same tables used in ANOVA. The 1 degree of freedom for the numerator associated with the mean that is being estimated will always be a value of 1 for confidence interval. The CI is used in this manner:

$$
\mu_{A 1}=\overline{A 1} \pm C I_{1}
$$

\section{RESULT AND DISCUSSION}

\section{A. Result}

THE EXPERIMENTAL RESULT IS SHOWN IN

Table 2. Scanning is applied three replication for each workpiece and dissimilarity calculated after conversion volume following mass into 230.385 (specimen with $1 \%$ vol. loss), 199.907 (specimen with 3\% vol. loss) and 196.032 (specimen with 5\% vol. loss). Data were measured in volume unit.

TABLE 2. EXPERIMENT RESULT

\begin{tabular}{|c|c|c|c|c|c|}
\hline \multirow{5}{*}{ 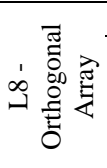 } & NO & 1 & 2 & 3 & 4 \\
\hline & $\mathrm{A}$ & 1 & 1 & 1 & 1 \\
\hline & B & 1 & 1 & 2 & 2 \\
\hline & $\mathrm{C}$ & 1 & 2 & 1 & 2 \\
\hline & $\mathrm{D}$ & 1 & 2 & 2 & 1 \\
\hline \multirow{4}{*}{$v_{s}$} & V1 $(1 \%)$ & 203.7353 & 204.919 & 203.334 & 201.9887 \\
\hline & V3 (3\%) & 197.5095 & 201.3073 & 199.1074 & 198.708 \\
\hline & V5 (5\%) & 196.1515 & 198.241 & 195.1159 & 197.8977 \\
\hline & Diss 1 & -0.172 & -0.754 & 0.025 & 0.687 \\
\hline \multirow[t]{2}{*}{$\psi$} & Diss 3 & 1.199 & -0.700 & 0.400 & 0.600 \\
\hline & Diss 5 & -0.061 & -1.127 & 0.467 & -0.952 \\
\hline \multirow{3}{*}{$\mathrm{S} / \mathrm{N}$} & $1 \%$ & 15.278 & 2.450 & 32.015 & 3.267 \\
\hline & $3 \%$ & -1.579 & 3.092 & 7.959 & 4.440 \\
\hline & $5 \%$ & 24.300 & -1.037 & 6.608 & 0.430 \\
\hline \multirow{5}{*}{ 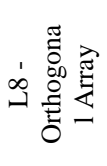 } & NO & 5 & 6 & 7 & 8 \\
\hline & $\mathrm{A}$ & 2 & 2 & 2 & 2 \\
\hline & B & 1 & 1 & 2 & 2 \\
\hline & $\mathrm{C}$ & 1 & 2 & 1 & 2 \\
\hline & D & 2 & 1 & 1 & 2 \\
\hline \multirow{3}{*}{$v_{s}$} & V1 $(1 \%)$ & 203.809 & 203.3472 & 202.3952 & 204.2011 \\
\hline & V3 (3\%) & 200.1078 & 200.3082 & 197.3087 & 200.3073 \\
\hline & V5 (5\%) & 196.2211 & 196.3456 & 194.2327 & 196.6779 \\
\hline \multirow{3}{*}{$\psi$} & Diss 1 & -0.208 & 0.019 & 0.487 & -0.401 \\
\hline & Diss 3 & -0.100 & -0.201 & 1.300 & -0.200 \\
\hline & Diss 5 & -0.096 & -0.160 & 0.918 & -0.329 \\
\hline \multirow{3}{*}{$\mathrm{S} / \mathrm{N}$} & $1 \%$ & 13.619 & 34.605 & 6.255 & 7.932 \\
\hline & $3 \%$ & 19.960 & 13.949 & -2.277 & 13.969 \\
\hline & $5 \%$ & 20.311 & 15.919 & 0.745 & 9.644 \\
\hline
\end{tabular}

Table 3 to Table 7 show the obtained results using ANOVA. The coefficient of determination is the ratio of the sum of squares of the predicted responses (corrected for the mean) to the sum of squares of the observed responses. This means that the mathematical model provides a good explanation of the relationship between the independent variables and volume scanned as a response. The associated p-value for the mesh is lower than 0.05 (i.e. $\alpha=0.05$, or $95 \%$ confidence) indicates that the model is considered to be statistically significant.

The results of the analysis of variance (ANOVA) conducted to determine the significant level of the variables on volume scanned (Table 3). P values which show the significance level of each variable and its degree of freedom (v), the sum of squares (SS), mean of squares (MS), F ratios and percentage of contribution Pc. Significance levels of the variables are determined by considering the F ratio in the ANOVA table. The results of ANOVA which was done to determine the significance level of the variable on the volume scanned. F ratios were taken into consideration to identify the significance level of the variables.

TABLE 3.

AVERAGE VALUE AND MAIN EFFECTS (RAW DATA) \& OPTIMAL FACTOR DISSIMILARITY $1 \%$

\begin{tabular}{cllllll}
\hline \hline Factor & \multicolumn{1}{c}{ SS } & $\mathbf{v}$ & $\mathbf{V}$ & \multicolumn{1}{c}{ F } & \multicolumn{1}{c}{ SS' $^{\prime}$} & Pc (\%) \\
\hline A & 0.00152 & 1 & 0.002 & 0.092 & -0.015 & -0.997 \\
\hline B & 0.45763 & 1 & 0.458 & 27.563 & 0.441 & 29.165 \\
C & 0.04225 & 1 & 0.042 & 2.545 & 0.026 & 1.696 \\
D & 0.69531 & 1 & 0.695 & 41.877 & 0.679 & 44.883 \\
Error & 0.3155 & 19 & 0.017 & & 0.38 & 25.253 \\
Total & 1.5122 & 23 & & & & 100.00 \\
\hline \hline
\end{tabular}

TABLE 4

ANOVA RESULTS FOR S/N RATIOS DISSIMILARITY 1\%

\begin{tabular}{cllllll}
\hline \hline Factor & \multicolumn{1}{c}{$\mathbf{S S}$} & $\mathbf{v}$ & \multicolumn{1}{c}{$\mathbf{V}$} & \multicolumn{1}{c}{$\mathbf{F}$} & \multicolumn{1}{c}{$\mathbf{S S}^{\prime}$} & \multicolumn{1}{c}{ Pc (\%) } \\
\hline A & 11.049 & 1 & 11.049 & 0.012 & -878.584 & -31.832 \\
B & 33.962 & 1 & 33.962 & 0.038 & -855.671 & -31.002 \\
C & 44.714 & 1 & 44.714 & 0.050 & -844.919 & -30.612 \\
D & 1.435 & 1 & 1.435 & 0.002 & -888.198 & -32.180 \\
Error & 2668.900 & 3 & 889.633 & & 6227.43 & 25.627 \\
Total & 2760.062 & 7 & & & & 100.00 \\
\hline \hline
\end{tabular}

TABLE 5

ANOVA RESULTS FOR RAW DATA OF DISSIMILARITY 3\%

\begin{tabular}{cllllll}
\hline \hline Factor & \multicolumn{1}{c}{ SS } & $\mathbf{v}$ & $\mathbf{V}$ & \multicolumn{1}{c}{ F } & \multicolumn{1}{c}{ SS' $^{\prime}$} & Pc (\%) \\
\hline A & 0.06129 & 1 & 0.061 & 1.415 & 0.018 & 0.425 \\
B & 0.45202 & 1 & 0.452 & 10.433 & 0.409 & 9.665 \\
C & 1.36144 & 1 & 1.361 & 31.424 & 1.318 & 31.172 \\
D & 1.53064 & 1 & 1.531 & 35.330 & 1.487 & 35.173 \\
Error & 0.8232 & 19 & 0.043 & & 1.00 & 23.565 \\
Total & 4.2286 & 23 & & & & 100.00 \\
\hline \hline
\end{tabular}

TABLE 6.

ANOVA RESULTS FOR S/N RATIOS OF DISSIMILARITY 3\%

\begin{tabular}{cllllll}
\hline \hline Factor & \multicolumn{1}{c}{ SS } & $\mathbf{v}$ & \multicolumn{1}{c}{$\mathbf{V}$} & $\mathbf{F}$ & \multicolumn{1}{c}{ SS' $^{\prime}$} & Pc (\%) \\
\hline A & 125.516 & 1 & 125.516 & 0.613 & -79.403 & -8.938 \\
B & 16.050 & 1 & 16.050 & 0.078 & -188.869 & -21.259 \\
C & 16.212 & 1 & 16.212 & 0.079 & -188.707 & -21.241 \\
D & 115.873 & 1 & 115.873 & 0.565 & -89.046 & -10.023 \\
Error & 614.757 & 3 & 204.919 & & 1434.43 & -38.539 \\
Total & 888.409 & 7 & & & & 100.00 \\
\hline \hline
\end{tabular}

TABLE 6.

ANOVA RESULTS FOR RAW DATA OF DISSIMILARITY 5\%

\begin{tabular}{cccclcl}
\hline \hline Factor & \multicolumn{1}{c}{ SS } & $\mathbf{v}$ & \multicolumn{1}{c}{ V } & \multicolumn{1}{c}{ F } & \multicolumn{1}{c}{ SS' $^{\prime}$} & Pc (\%) \\
\hline A & 0.50207 & 1 & 0.502 & 13.733 & 0.466 & 13.758 \\
B & 0.29962 & 1 & 0.300 & 8.195 & 0.263 & 7.775 \\
C & 1.80095 & 1 & 1.801 & 49.259 & 1.764 & 52.146 \\
D & 0.08625 & 1 & 0.086 & 2.359 & 0.050 & 1.469 \\
Error & 0.6947 & 19 & 0.037 & & 0.84 & 24.852 \\
Total & 3.3836 & 23 & & & & 100.00 \\
\hline \hline
\end{tabular}

TABLE 7.

ANOVA RESULTS FOR S/N RATIOS OF DISSIMILARITY 5\%

\begin{tabular}{clllccl}
\hline \hline Factor & \multicolumn{1}{c}{ SS } & $\mathbf{v}$ & \multicolumn{1}{c}{$\mathbf{V}$} & \multicolumn{1}{c}{ F } & \multicolumn{1}{c}{ SS' $^{\prime}$} & Pc (\%) \\
\hline A & 33.289 & 1 & 33.289 & 0.096 & -315.032 & -22.584 \\
B & 221.210 & 1 & 221.210 & 0.635 & -127.111 & -9.112 \\
C & 91.180 & 1 & 91.180 & 0.262 & -257.141 & -18.434 \\
D & 4.305 & 1 & 4.305 & 0.012 & -344.017 & -24.662 \\
Error & 1044.964 & 3 & 348.321 & & 2438.25 & -25.209 \\
Total & 1394.948 & 7 & & & & 100.00 \\
\hline \hline
\end{tabular}




\section{B. Discussion}

Average volume scanned was measured after the experiments performed according to the L8 orthogonal array. The experimental results and $\mathrm{S} / \mathrm{N}$ ratios calculated according to Taguchi's "the-smaller-the-better" quality characteristic were given in Table 3 - Table 6 . The quality of dissimilarity volume scanned was evaluated in terms of dissimilarity Diss 1, Diss 3, and Diss 5. For this purpose, graphs are given in Figure 2. Main effect plot has been constructed to illustrate the relationships between volume scanned and scanning parameters.

TABLE 8 .

AVERAGE VALUE AND MAIN EFFECTS (RAW DATA) \& OPTIMAL FACTOR DISSIMILARITY $1 \%$

\begin{tabular}{ccclc}
\hline \hline Level & Shut. Time & Grid & Distance & Mesh \\
\hline 1 & -0.054 & -0.279 & 0.033 & 0.255 \\
2 & -0.026 & 0.199 & -0.113 & -0.335 \\
\multicolumn{4}{c}{ Optimal level (Lower is the better) } & Average value \\
& A1 & -0.054 \\
B1 & & -0.279 \\
C2 & -0.113 \\
D2 & -0.335 \\
\hline \hline
\end{tabular}

TABLE 9

Average VALUE AND MaIN EFFECTS (RAW DATA) \& OPTIMAL FACTOR DISSIMILARITY 3\%

\begin{tabular}{cllll}
\hline \hline Level & Shut. Time & Grid & Distance & Mesh \\
\hline 1 & 0.375 & 0.049 & 0.700 & 0.725 \\
2 & 0.200 & 0.525 & -0.125 & -0.150 \\
& Optimal factor (Lower is the better) \\
Optimal level & \multicolumn{3}{c}{ Average value } \\
A1 & \multicolumn{2}{c}{0.375} \\
B1 & 0.049 \\
C2 & -0.125 \\
& D2 & -0.150 \\
\hline \hline
\end{tabular}

TABLE 10.

AVERAGe VAlue AND MAIN EFFECTS (RAW DATA) \& OPTIMAL FACTOR DISSIMILARITY $5 \%$

\begin{tabular}{ccccc}
\hline \hline Level & Shut. Time & Grid & Distance & Mesh \\
\hline 1 & -0.418 & -0.361 & 0.307 & -0.064 \\
2 & 0.083 & 0.026 & -0.642 & -0.150 \\
Optimal level (Lower is the better) & Average value \\
& A1 & & -0.418 \\
& B1 & -0.361 \\
C2 & & -0.642 \\
D2 & -0.150 \\
\hline \hline
\end{tabular}

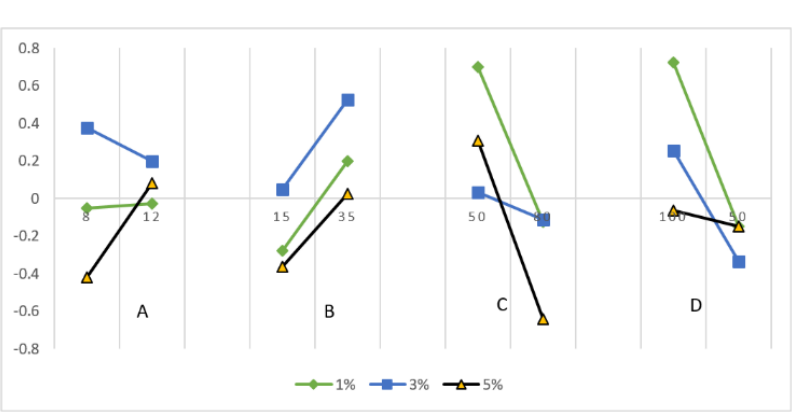

Figure 2. Main effect plot.

IN TABLE 4, TABLE 6.

ANOVA RESULTS FOR S/N RATIOS OF DISSIMILARITY 3\%

and Table 7 shows $\mathrm{S} / \mathrm{N}$ have less value compared to the percent contribution of parameter studied (see Table 3, Table
5, Table 6). Hence, the experiment concerned about raw data scanning. Figure 2 shows high factor prompting in volume scan. Dissimilarity at $1 \%$ volume loss would be optimum for following parameters A2, B2, C1, D1. Dissimilarity at 3\% volume loss would be optimum for following parameters A1, B2, C1, D1. Finally, dissimilarity at $5 \%$ volume loss would be optimum for following parameters A2, B2, C1, D1. The trend line for volume scan measurement would be steady at $5 \%$ volume loss. Since volume scan at 5\% approaching zero, this value indicated the ability of micro wear measurement recently.

FCBT which are the specimens used in this study suffering polishing/grind wear mechanism. They have a smooth and shiny surface. However, a further study regarding the form of scar in the worn component which is a rough surface should be carried out in the future.

\section{Estimation of Optimum Performance Characteristics}

The estimated mean value of the response characteristic, dissimilarity volume was determined as follows. The mean values of all the quality characteristics at the optimum levels of significant factors are presented in Table 8 to Table 10. The optimal values of the predicted means $(\mu)$ of different response characteristics in every single percentage of dissimilarity volume can be obtained from the following formula:

$$
\begin{aligned}
& \mu_{1 \%}=A 2+B 2+C 1+D 1-3 T \\
& \mu_{3 \%}=A 1+B 2+C 1+D 1-3 T \\
& \mu_{5 \%}=A 2+B 2+C 1+D 1-3 T
\end{aligned}
$$

The $95 \%$ confidence interval of confirmation experiments $\left(C I_{\% \text { diff }}\right.$ ) can be computed by using the following Equation:

$$
C I_{\% \operatorname{dissVL}}=\sqrt{F_{\alpha}\left(1, f_{e}\right) V_{e}\left[\frac{1}{n_{e f f}}+\frac{1}{R}\right]}
$$

For dissimilarity volume loss,

$$
\begin{gathered}
\mu_{1 \%}=-0.026+0.199+0.700+0.725+0.120 \\
=1.717 \\
\mu_{3 \%}=0.375+0.525+0.033+0.255-0.861=0.326 \\
\mu_{5 \%}=0.083+0.026+0.307-0.064+0.503=0.855
\end{gathered}
$$

From equation of $C I_{\%}$ dissVL

$$
\begin{aligned}
& C I_{1 \%}= \pm 0.0069 \\
& C I_{3 \%}= \pm 0.0111 \\
& C I_{5 \%}= \pm 0.0102
\end{aligned}
$$

The predicted optimal limit for three times of experiments is given by

$$
\begin{aligned}
& C I_{1 \%}=1.724<\mu<-1.710 \\
& C I_{3 \%}=0.337<\mu<-0.315 \\
& C I_{5 \%}=0.865<\mu<-0.845
\end{aligned}
$$

The optimal values of dissimilarity volume loss were predicted at the selected levels of significant factors.

$\mu_{1 \%}=$ Shutter time $=12 \mathrm{~ms} ;$ Grid $=35 ;$ Distance $=50 ;$ Mesh $=100$ (optimal parameter for dissimilarity volume loss $1 \%$ ) 
$\mu_{3 \%}=$ Shutter time $=8 \mathrm{~ms} ;$ Grid $=35 ;$ Distance $=50 ;$ Mesh

$=100$ (optimal parameter for dissimilarity volume loss $3 \%$ )

$\mu_{5 \%}=$ Shutter time $=12 \mathrm{~ms} ;$ Grid $=35 ;$ Distance $=50 ;$ Mesh

$=100$ (optimal parameter for dissimilarity volume loss $5 \%)$

\section{CONCLUSION}

In this study, the statistical methods of signal-to-noise $(\mathrm{S} / \mathrm{N})$ ratio and the analysis of variance (ANOVA) are applied to find out an optimal composition of several parameters for capturing 3D imaging of wear volume in a worn bucket teeth sample. This study conclude that:

1. The Taguchi robust design method explains that among the four factors and levels tested, all factors show a significant response.

2. The effects of the variables on volume dissimilarity were determined by the ANOVA. The most significant variable for $1 \%$ volume loss was found the focus range distance $(50 \mathrm{~mm})$ and meshing method (100) while the variable that was the most significant for $3 \%$ and $5 \%$ volume loss was the meshing method.

3. The parameter studied to provide the micro wear ability at $5 \%$ volume loss since dissimilarity obtained approaching to the highest value of similarity.

4. The ANOVA results show that the optimum conditions are $12 \mathrm{~ms}$ of shutter time (A2), $15 \mathrm{~mm}$ of the distance between the grid (B2), $50 \mathrm{~mm}$ of the distance to capture
(C1), and normal mesh (D1) as a method to polygonize the point clouds.

5. All values of dissimilarity volume loss had been validated by confirmation of experiments and being accepted in between the predicted optimal limit.

\section{ACKNOWLEDGMENT}

This study is supported by P3MI ITB 2017 the authors express appreciation for this support.

\section{REFERENCES}

[1] H. Zum Gahr and G. T. Eldis, "Abrasive wear of white cast irons," Wear, pp. 175-194, 1980.

[2] J. Coronado, A. Gómez, and A. Sinatora, "Tempering temperature effects on abrasive wear of mottled cast iron," Wear, vol. 267, pp. 2070-2076, 2009.

[3] M. Mashloosh and T. S. Eyre, "Abrasive wear and its application to digger teeth," Tribol. Int., vol. 18, pp. 259-266, 1985.

[4] Anonymous, "Penelitian Keausan Gigi BWD dan Upaya Modifikasi Untuk Meningkatkan Umur Efektifnya," Bandung, 2011.

[5] K. Lee, Principles of CAD/CAM/CAE systems. Addison-Wesley, 1999.

[6] M. Daoudi, A. Srivastava, and R. Veltkamp, 3D Face Modeling, Analysis and Recognition. Wiley, 2013.

[7] P. J. Ross, Taguchi techniques for quality engineering: loss function, orthogonal experiments, parameter and tolerance design. New York: McGraw-Hill, 1996.

[8] V. Raja and K. J. Fernandes, Reverse Engineering: An Industrial Perspective. London: Springer, 2007. 